\title{
Pitutaty insufficiency. Diagnosis masked by a toxic thyroid adenoma
}

\author{
Maria Alevizaki $^{1,2}$, Lia-Angela Moulopoulos ${ }^{3}$, Emily Mantzos ${ }^{1}$, Demetrios A. Koutras ${ }^{1,2}$ \\ Endocrine Units, "Evgenidion" Hospital ${ }^{1}$, Department of Clinical Therapeutics", ALEXANDRA Hospital, and ${ }^{3}$ Dept \\ of Roentgenology, Athens University School of Medicine, 11528 Athens, Greece
}

\begin{abstract}
A patient with undiagnosed pituitary insufficiency who also had a toxic thyroid adenoma is presented. The T3 secreted by the adenoma apparently corrected the secondary thyroid failure, but also aggravated the secondary adrenal failure and almost precipitated adrenal crisis. The diagnostic work-up in such an unusual co-existence is discussed, in particular since the clinical diagnosis of pituitary insufficiency in the elderly is not easily established
\end{abstract}

Key words: Pituitary insufficiency, empty sella, toxic adenoma.

\section{INTRODUCTION}

The diagnosis of a toxic uninodular goiter or toxic adenoma is straightforward in the case of a single "hot" nodule which presents with a high T3 (sometimes also T4) and low TSH levels. The diagnosis of pituitary insufficiency is not particularly easy in persons beyond the reproductive age when loss of libido and facial hair in men or loss of menstruation in women may go unrecognized. The diagnosis is further complicated when toxic thyroid adenoma and pituitary insufficiency occur concomitantly in a postmenopausal woman. The evaluation of such a case and the difficulties involved are underlined by the present case.

Address correspondence and requests for reprints to: Demetrios A. Koutras, 35, Vassilissis Sofias Ave, 10675 Athens, Greece, Phone -3010 - 7211319, FAX -3010-7246 003, e-mail: mani@otenet.gr

Received 5-4-02, Revised 27-5-02 Accepted 6-6-02

\section{CASE HISTORY}

A 67-year old woman was referred to one of us (D.A.K.) for evaluation of her thyroid function. She complained of weight loss and extreme fatigue of two months duration. Her blood pressure was reported as low. Repeated examinations had shown low or lownormal T4 and FT4 values, high normal or frankly high T3 and FT3 values, whereas serum TSH had always been very low. An ultrasonogram had shown a single solid nodule with a maximum diameter of $17 \mathrm{~mm}$ in the left lobe of the thyroid. This nodule was hot in the scintigram (Figure 1), whereas the rest of the thyroid parenchyma was suppressed. Routine hematologic parameters were within the normal range except for mild lymphocytosis and eosinophilia. The physicians who had previously examined the patient had refrained from any treatment.

On inspection the patient appeared pale, drowsy and debilitated. She could walk only with the help of her daughter, who reported that the patient had last given birth at the age of 27 years and that her menses 


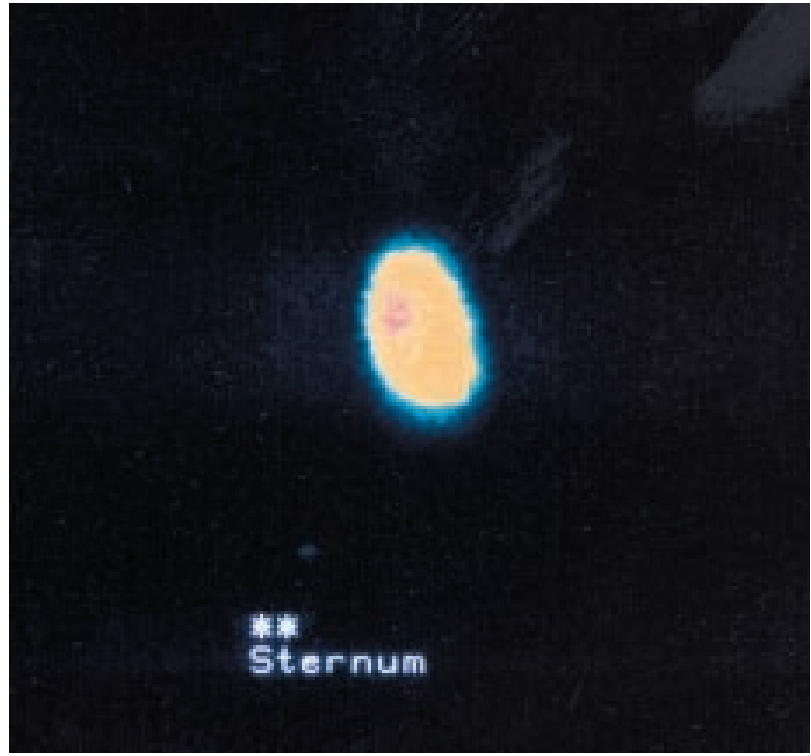

Figure 1. Autonomous thyroid nodule resulting in "self cure" of pituitary hypothyroidism and unmasking of a simultaneously present pituitary insufficiency.

had continued normally until the age of 50 years. The patient's hands were cold. The pulse rate was $96 / \mathrm{min}$, blood pressure 125/75 mm Hg, body weight $65.3 \mathrm{~kg}$, height $154 \mathrm{~cm}$. Pubic and axillary hair were absent. The appearance of the patient suggested pituitary insufficiency, which led to further endocrine work-up. Serum $\mathrm{T}_{4}$ was $77.2 \mathrm{nmol} / \mathrm{L}$ (normal $69.5-161 \mathrm{nmol} / \mathrm{L}$ ), $\mathrm{T}_{3} 3.04 \mathrm{nmol} / \mathrm{L}$ (normal 0.92-2.61 nmol/L), $\mathrm{RT}_{3} \mathrm{U}$ $27.3 \%$ (normal 25\%-35\%), free-thyroxine index 4.5 (normal 9-25), TSH $0.01 \mu \mathrm{U} / \mathrm{ml}$. TSH receptor antibodies (TRAK) were low $(0.4 \mathrm{U} / \mathrm{L})$. Basal serum cortisol was $85 \mathrm{nmol} / \mathrm{L}$ (normal 193-607 nmol/L), ACTH $4.8 \mathrm{pg} / \mathrm{ml}$ (normal $9-42 \mathrm{pg} / \mathrm{ml}$ ) and the $24 \mathrm{~h}$ urinary cortisol was low $(30 \mathrm{nmol} / 24 \mathrm{~h})$. Despite the postmenopausal state, the gonadotrophin levels were low and did not respond to LHRH stimulation $\left(\mathrm{LH}_{0}=2.1\right.$, $\left.\mathrm{LH}_{30}=3.4, \mathrm{FSH}_{0}=1.5, \mathrm{FSH}_{30}=1.6 \mu \mathrm{U} / \mathrm{ml}\right)$. Basal prolactin levels were low $(6.5 \mathrm{ng} / \mathrm{ml})$ and did not respond to TRH stimulation, while GH levels were undetectable. Blood glucose was $57 \mathrm{mg} / \mathrm{dl}$. Computerised axial tomography of the pituitary was suggestive of empty sella (Figure 2). The MRI showed the existence of normal pituitary tissue but of reduced size.

A diagnosis of pituitary insufficiency was established and hydrocortisone was prescribed at $20 \mathrm{mg}$ daily. Two days later the patient's condition had strik-

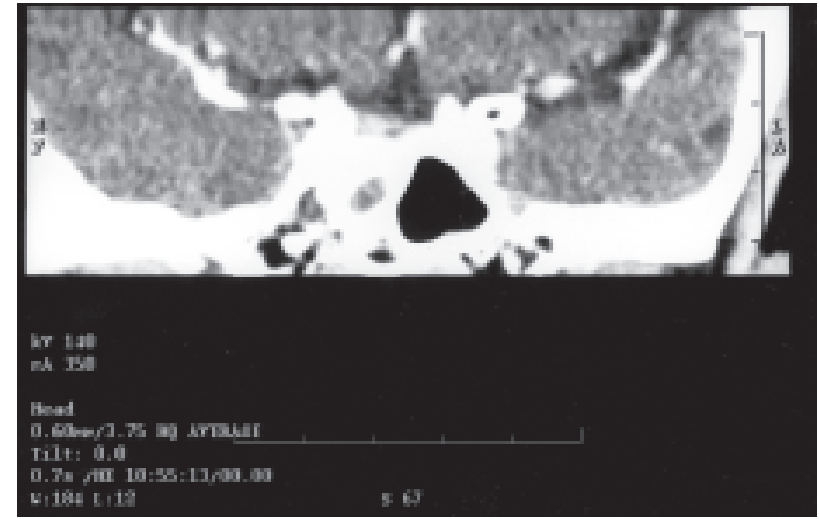

Figure 2. CT scan of the pituitary gland showing signs of partially empty sella. The normal pituitary reduced in size is shown on the right side of the sella

ingly improved. When seen again after two months, her appearance had changed completely. She walked briskly, smiling and with confidence into the consulting room, expressing her thanks for her improvement and said that she felt stronger and healthier. Laboratory investigations this time showed: serum $\mathrm{T}_{4} 86.2$ $\mathrm{nmol} / \mathrm{L}, \mathrm{T}_{3} 1.59 \mathrm{nmol} / \mathrm{L}$ and $\mathrm{TSH} 0.01 \mu \mathrm{U} / \mathrm{ml}$.

\section{DISCUSSION}

Silent pituitary insufficiency masked by a toxic adenoma of the thyroid has not previously been reported. "Self cure" of pituitary hypothyroidism has been reported in cases in which autoimmune hyperthyroidism developed in patients with pituitary insufficiency, usually secondary to a pituitary tumour ${ }^{1,2}$. A theoretical possibility linking pituitary insufficiency with hyperthyroidism could be autoimmune hypophysitis coexisting with autoimmune hyperthyroidism. However, in our patient TRAK were negative and the clinical and radionuclide scan findings were consistent with an autonomous nodule.

The relative decrease of $\mathrm{T} 3$ levels during followup cannot be easily explained; a possible transient increase in iodine intake through an unknown source could, in theory, explain the previously elevated levels. Another possibility might be that the correction of secondary adrenal insufficiency with corticosteroids may have decreased the conversion of $\mathrm{T} 4$ to $\mathrm{T} 3$. Alternatively, a spontaneous decrease in the functional activity of the hot nodule constitutes another possibility. Transient pharmacological effect of the iodine 
contained in the contrast medium used for the CT scan might, in theory, explain the reduction of thyroid hormone synthesis and release by the hot nodule. However, such an effect would be expected to be only transient. The diagnosis of primary thyroid disorders (ex. primary hypothyroidism) is always difficult in the presence of pituitary insufficiency and has to be based on peripheral thyroid hormone levels.

To assess pituitary function in an adult, hypothalamic releasing hormones may be used: LHRH for gonadotrophin reserve, TRH for TSH and PRL response, GHRH for GH and CRH for ACTH. The latter two hormones may be assessed simultaneously with the insulin tolerance test, which has the advantage of assessing hypothalamic as well as pituitary function so far as ACTH and GH are concerned; this test was, however, not performed because of the age and the poor health of the patient ${ }^{3}$. A CRH test, on the other hand, would have been quite helpful but it was unfortunately not available at the time the patient was evaluated. Nevertheless, it has been suggested that in the absence of previous corticosteroid administration, low cortisol levels associated with low ACTH are strongly suggestive of secondary adrenal failure and that further work-up is not necessary ${ }^{4}$. A single GHRH test may not be very informative for GH reserve, especially in an elderly patient, and frequently needs to be repeated ${ }^{4}$. The patient's pituitary insufficiency was thus documented for gonadotrophins (inappropriately low for a postmenopausal woman and not responding to LHRH) and prolactin, and only indirectly for ACTH, through very low basal cortisol and ACTH levels.

The cause of the pituitary insufficiency in our patient is not obvious. One possibility might be that it was autoimmune in nature ${ }^{4}$; unfortunately, we did not have the opportunity to determine pituitary autoantibodies. The most common type, lymphocytic hypophysitis, is encountered predominantly in younger women during late pregnancy or early post-partum and in the vast majority of cases an enlarged pituitary and thickening of the stalk as well as loss of the bright spot of the posterior pituitary are seen on the MRI ${ }^{4}$. Diabetes insipidus may coexist and usually there is evidence of other autoimmune disorders such as lymphocytic thyroiditis; ; none of these findings were present in our patient. Infiltration by a granulomatous disease or metastases to the pituitary also tend to present with diabetes insipidus and result in an enlarged gland ${ }^{4}$. Autoimmune partial ACTH insufficiency accompanied by autoimmune thyroid failure, which was reversible after correction with glucocorticoids, has been reported in a case of empty sella syndrome ${ }^{6}$.

Another possibility is that a preexisting pituitary adenoma underwent hemorrhage and necrosis. When asked, the patient recalled that 2 years previously she had suddenly felt very bad and had fainted and vomited. One cannot therefore entirely exclude the possibility of necrosis of a pituitary tumour, though MRI did not show any evidence for this. In any case, the pituitary insufficiency must have occurred relatively recently.

Generalized pituitary insufficiency is easily suspected in children suffering from dwarfism, and in persons of reproductive age suffering from hypogonadism. In elderly people, however, lack of energy and reduced pubic and axillary hair may be attributed to age. The diagnosis may be even less apparent in the unusual simultaneous occurrence of an autonomous adenoma of the thyroid, which "cures" the secondary hypothyroidism caused by pituitary insufficiency. In the event of such a coexistence, high T3 levels are not compatible with pituitary insufficiency and low TSH levels are attributed to the toxic adenoma. Pituitary insufficiency may thus be overlooked.

In our case, the high level of circulating T3 may have increased the requirements for glucocorticoids and precipitated the secondary adrenal insufficiency ${ }^{7}$, which led the patient to seek medical assistance. Our coclusion is that, should the physician try to explain clinical findings on the basis of a single disorder, the diagnosis of the rare occurrence of two separate ones may be delayed or elude detection.

\section{REFERENCES}

1. Otsuka F, Ogura T, Hayakawa N, Mimura Y, Kageyama J, Makino H, 1997 Manifestation of primary hyperthyroidism after pituitary adenomectomy: a case report. Endocr J 44: 887-893.

2. Krishnamurthy GT, Blahd WH, 1974 Hyperthyroidism in the presence of panhypopituitarism. Thyroid crisis and hypothyroidism following radiodine treatment. West $\mathbf{J}$ Med 120: 491-496.

3. Werbel SS, Ober PK, 1993 Acute adrenal insufficiency: diagnostic testing. Endocr Metab Clin North Am 22: 303328.

4. Lee Vance M, Hypopituitarism, 1994 N Engl J Med 330: 
$1651-1662$

5. Cheung CC, Ezzat S, Smyth HS, Asa SL, 2001 The spectrum and significance of primary hypophysitis. J Clin Endocrinol Metab 86: 1048-1053.

6. Otsuka F, Ogura T, Hayakawa N, Harada S, Kageyama J,
Makino H, 1998 Reversible hypothyroidism in empty sella syndrome: a case report. Endocr J 45: 385-391.

7. Murray JS, Jayarajasingh R, Perros P, 2001 Lesson of the week: Deterioration of symptoms after start of thyroid hormone replacement. BMJ 23: 332-333. 